\title{
Does pulmonary surfactant generally affect antimicrobial activity?
}

\author{
Richard Schwameis, Sabine Strommer, Markus Zeitlinger, Robert Sauermann ${ }^{*}$ \\ From 18th Scientific Symposium of the Austrian Pharmacological Society (APHAR). Joint meeting with the \\ Croatian, Serbian and Slovenian Pharmacological Societies. \\ Graz, Austria. 20-21 September 2012
}

\section{Background}

Activity of antimicrobial agents may be affected by pulmonary surfactant. Notably, it was reported that the clinical efficacy of daptomycin is significantly impaired in pneumonia in spite of bacterial susceptibility in vitro. This study set out to assess the impact of pulmonary surfactant in vitro on bacterial killing of other antibiotics used for treatment of pneumonia.

\section{Methods}

Time-kill curves of daptomycin, doripenem, linezolid, moxifloxacin, and tigecycline were determined for Staphylococcus aureus ATCC 29213 and of colistin, doripenem and moxifloxacin for a clinical isolate of Pseudomonas aeruginos $a$ at concentrations above or equal to the respective MICs. All experiments were performed over $24 \mathrm{~h}$ in Mueller-Hinton broth (MHB) and in MHB enriched with porcine surfactant at a concentration of $1 \mathrm{mg} / \mathrm{mL}$ ( $\left.\mathrm{MHB}_{\text {surf }}\right)$.

\section{Results}

As expected, daptomycin was not bactericidal in presence of surfactant at concentrations up to 64 times the MIC. In $\mathrm{MHB}_{\text {surf }}$ a higher concentration of moxifloxacin (16x) was needed than in MHB (2x MIC) to achieve sustained bacterial killing of $S$. aureus. In contrast, killing of $P$. aeruginosa by moxifloxacin was not affected by surfactant. A slightly higher concentration of doripenem (8x) was needed in $\mathrm{MHB}_{\text {surf }}$ to achieve sustained antimicrobial killing against $S$. aureus than in MHB (4x MIC). However, killing was faster in $\mathrm{MHB}_{\text {surf }}$. Similarly, initial killing of $S$. aureus by tigecycline was faster in $\mathrm{MHB}_{\text {surf }}$ than in MHB while after 24 hours no difference in bacterial counts was observed between MHB and $\mathrm{MHB}_{\text {surf. }}$ For linezolid no significant effects were observed by adding surfactant. Likewise, surfactant had no significant influence on the activity of colistin and doripenem against $P$. aeruginosa.

\section{Conclusions}

The activity of moxifloxacin against $S$. aureus was reduced in vitro by addition of surfactant whereas this effect could not be observed against $P$. aeruginosa. Interestingly, antimicrobial killing by several antibiotics of Gram-positive $S$. aureus, but not of Gram-negative P. aeruginosa tended to be faster in presence of surfactant. Thus, apart from daptomycin, pulmonary surfactant is also capable of influencing the bacterial killing kinetics of several other antibiotics. The clinical relevance of these in vitro findings for pneumonia patients is currently unclear, and should be carefully evaluated.

Published: 17 September 2012

doi:10.1186/2050-6511-13-S1-A62

Cite this article as: Schwameis et al.: Does pulmonary surfactant generally affect antimicrobial activity? BMC Pharmacology and Toxicology 2012 13(Suppl 1):A62.

\footnotetext{
* Correspondence: robert.sauermann@meduniwien.ac.at Department of Clinical Pharmacology, Section of Clinical Pharmacokinetics, Pharmacogenetics and Imaging, Medical University of Vienna, 1090 Vienna, Austria
}

(c) 2012 Schwameis et al; licensee BioMed Central Ltd. This is an Open Access article distributed under the terms of the Creative Commons Attribution License (http://creativecommons.org/licenses/by/2.0), which permits unrestricted use, distribution, and reproduction in any medium, provided the original work is properly cited. 